\title{
Theoretical and Experimental Study on Sound Absorption of a Multi-leaf Microperforated Panel*
}

\author{
I Made MIASA $^{* *}$ and Masaaki OKUMA ** \\ **Department of Mechanical \& Aerospace Engineering, Tokyo Institute of Technology \\ 2-12-1 O-okayama, Meguro-ku, Tokyo 152-8552, JAPAN \\ email': miasa@stu.mech.titech.ac.jp \\ email': mokuma@mech.titech.ac.jp
}

\begin{abstract}
A theoretical method is presented for calculating the absorption coefficient of a multi-leaf microperforated panel absorber. In this work, the possibility of using multi-leaf type of microperforated panels without solid backing panel is investigated. The number of panels varies from 2 to $n(\geq 2)$. The sound absorption coefficient is calculated based on electro-acoustic analogy by taking the sound transmission coefficient due to the absence of solid backing panel into consideration. The optimum performance of leaf microperforated panel absorber is also investigated as the function with respect to the design parameters such as perforation ratio, diameter of holes and distance between panels. The numerical results show that the configuration of leaf of microperforated panels can increase absorption coefficient at low frequency region. This fact will give an advantage to the multi-leaf microperforated panel over conventional microperforated panel arrangements. That is, to solve the problem of quite narrow frequency band absorption effect, especially in mid-high frequency region corresponding to their resonant frequencies, that the conventional microperforated panel arrangements inherently possess. Experiment results are also presented that show a reasonable agreement with the theoretical results. Some feasible application ideas of the multi-leaf type of arrangement are also presented.
\end{abstract}

Key words: Sound Absorption Coefficient, Multi-leaf Microperforated Panel, Solid Backing Panel, Conventional Microperforated Panel Absorber, Open Air

\section{Introduction}

Recently the use of Microperforated Panel Absorber (MPA) as an alternative to the conventional absorber is increasing. It has been recognized as a fiber-free absorber and for its attractive appearance. The story of MPA started when Maa [1,2,3] first derived the formulas used for MPA and then investigated the potential of MPA. Since then its application, improvement and theoretical development of MPA have been studied intensively $[4,5,6,7,8]$. In all of the previous works dealing with MPA, the conventional arrangement of microperforated panel (MPP) is considered, until Sakagami et al. [9] introduced the double leaf MPP as another type of sound absorber. Conventional means that MPP arrangement has to be terminated with a hard wall. In this work, the number of panel is extended into $n$ number of panel, and the characteristics of each arrangement are discussed and then verified with some measurements. Optimum design of this absorber is also investigated, and in addition, some feasible application ideas are also presented. 


\section{Basic theory of MPA}

The MPP absorbers have a simple structure and absorption characteristics that are exactly predictable. It consists of panel perforated with sub millimeter orifices in front of an air cavity. The panel may be made of any material such as cardboard, plastic, sheet of metal or wood. Appropriately choosing the parameters of the MPP, absorption in certain frequency bands can be obtained using a cavity of a moderate thickness without the need of additional fibrous material. This will provide a clean-washable absorbent system.

An MPP can be made with distribution of narrow short tubes with hole's distance that is small compared to the wavelength of the impinging sound wave. The propagation of a sound wave in a tube was first studied by Lord Rayleigh and then simplified for short tubes by Crandall (see [2,3]). The equation of aerial motion in a tube short compared to the wavelength is given by

$$
j \omega \rho_{0} u-\frac{\eta}{r_{1}} \frac{\partial}{\partial r_{1}}\left[r_{1} \frac{\partial}{\partial r_{1}} u\right]=\frac{\Delta P}{t},
$$

where $\Delta p$ is the sound pressure difference between the ends of the tube, $t$ is the length of the tube (or the thickness of the panel), $\rho_{0}$ is the density of air, $\eta$ is the coefficient of viscosity, and $r_{1}$ is the radius of the holes. The equation may be solved for the particle velocity $u$, and the ratio of $\Delta p$ to the average value of $\bar{u}$ over the cross-sectional area of the tube gives the specific acoustic impedance of the short tube as follows

$$
Z_{1}=\frac{\Delta p}{\bar{u}}=j \omega \rho_{0} t\left[1-\frac{2}{k \sqrt{-j}} \frac{J_{1}(k \sqrt{-j})}{J_{0}(k \sqrt{-j})}\right]^{-1},
$$

where $k=d / \sqrt{4 \eta / \rho_{0} \omega}$ is the perforate constant defined as the ratio of the orifice diameter, $d$, to the viscous boundary layer thickness of the air in the orifice. $J_{0}$ and $J_{1}$ are the Bessel functions of the first kind of orders zero and one, respectively and $\omega$ is the angular frequency.

The sound absorption mechanism of MPA mainly occurs due the effects of the micro-perforation and the resonance phenomena the same with the phenomena found in Helmholtz resonator. The perforations, holes or slots with air space behind them will act as multiple cavity resonators, and the micro size of the perforation will give high sound resistance due to the viscous boundary layers effects in the perforations so that additional sound absorber is not needed anymore as of the conventional Helmholtz resonator. The cavity and neck or perforations combination resemble a spring mass combination and as such have a resonant frequency determined by the mass of the air in the neck and the springiness of the trapped air in the cavity depth. At resonant and neighboring frequencies, the air or sound wave moves vigorously in and out of the cavity causing the acoustic energy of the air to be converted into heat and dissipated. The maximum sound absorption will occur around the resonant frequency and quite wide-band sound absorption around the resonance occurs due the low acoustic mass reactance of the perforations. Obviously the amount of the heat generated is very small as the sound energy is also quite small if one considers it in watts. Another mechanism that could enhance the energy loss in this kind of construction is the fact that the surface of the panel is not smooth anymore because of many holes on it. The edge effect of the holes also in some extend will re-route the sound wave, some will be reflected back out of phase so that small cancellation is also produced just in front of the panel.

Maa [1] introduced approximation formula for Eq. (2) that holds for all value of $k$ and included the end correction factor as

$$
Z_{1}=R+j X
$$

where 


$$
\begin{aligned}
& R=\frac{32 \eta t}{d^{2}}\left[\left(1+\frac{k^{2}}{32}\right)^{1 / 2}+\frac{\sqrt{2}}{32} k \frac{d}{t}\right], \\
& X=\operatorname{\omega t\rho }_{o}\left(1+\left[1+\frac{k^{2}}{2}\right]^{-1 / 2}+0.85 \frac{d}{t}\right),
\end{aligned}
$$

where $R$ and $X$ are resistance and reactance part of the hole impedance respectively that holds for all value of $k$. The porosity of panel or perforation ratio $(\sigma)$ is ratio between total area of holes and total area of panel that is

$$
\sigma=0.785\left(d^{2} / b^{2}\right) \text {, }
$$

where $b$ is the separation distance between holes.

The following equation is used for the specific acoustic impedance of the MPP when normalized by the air characteristic acoustic impedance $\left(Z_{0}=\rho_{o} c\right)$ and porosity of panel $(\sigma)$

$$
z_{m p p}=Z_{1} /\left(\sigma \rho_{0} c\right)=r+j x_{m}=r+j \omega m,
$$

where $r$ and $x_{m}$ are resistance and reactance part of the panel impedance respectively, $m$ is the acoustic mass; $c$ is speed of sound.

MPP placed at a certain distance from a solid wall will make it a good sound absorber. The acoustic impedance of air cavity with a depth of $D$ behind MPP, normalized by the air characteristic acoustic impedance, is

$$
z_{\text {cavity }}=-j \cot \left(\frac{\omega}{c} D\right) \text {. }
$$

The overall acoustic impedance of MPP and air cavity arrangement will be

$$
z_{\text {total }}=z_{\text {mpp }}+z_{\text {cavity }} \text {. }
$$

Finally the sound absorption coefficient of the conventional MPA is calculated using the following well-known equation

$$
\alpha_{\text {con-MPA }}=1-\left|\frac{z_{\text {total }}-1}{z_{\text {total }}+1}\right|^{2} .
$$

\section{Electro-acoustic analog circuit}

An arrangement of $n$ number of panel is described in Fig. 1. The numbering for each panel is started from the right to the left. The distances between two adjacent panels from right to the left are $D_{2} \ldots D_{n}$ consecutively.

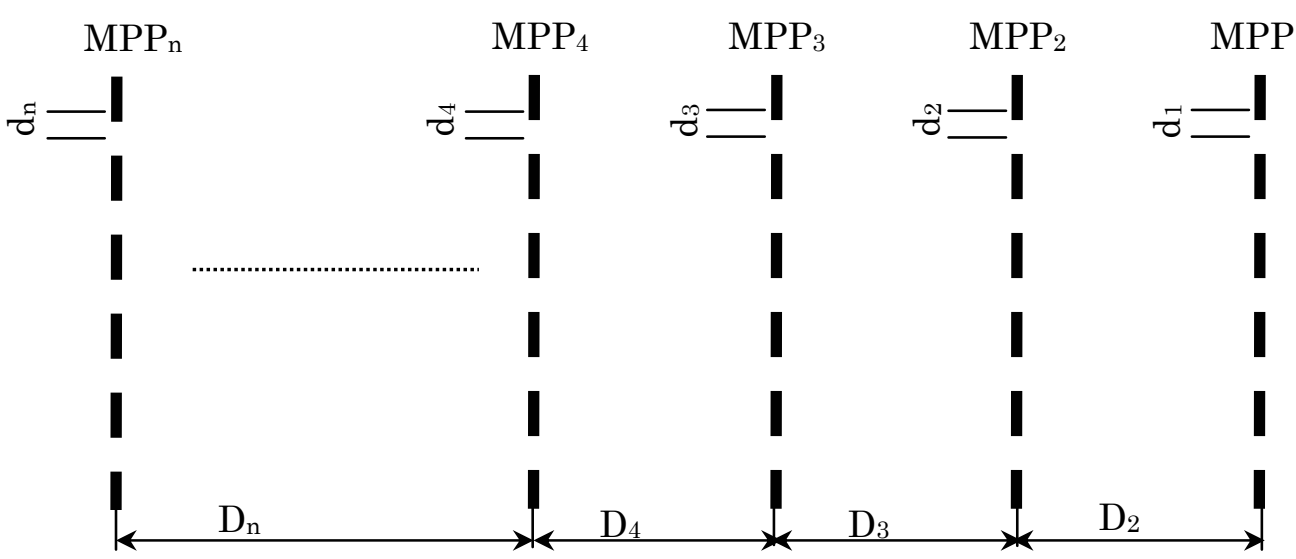

Figure 1. Microperforated panel arrangement 
From this configuration one can derive its corresponding electro-acoustic analog circuit as shown in Fig. 2. The impedance of the open air at far end of the circuit is written as $Z_{D 1}$ which is equal to the impedance of the open air just in front of panel number $n(M P P n)$ that is $Z_{D 1}=Z_{\text {open-air }}=Z_{0}=\rho_{o} c$. The impedance of each MPP consists of real part and imaginary part. The distances between panels $D_{2} \ldots D_{n}$ have impedances of $Z_{D 2} \ldots Z_{D n}$ respectively. A plane sound wave of unit pressure amplitude is assumed to be normally incident upon MPP $_{n}$.

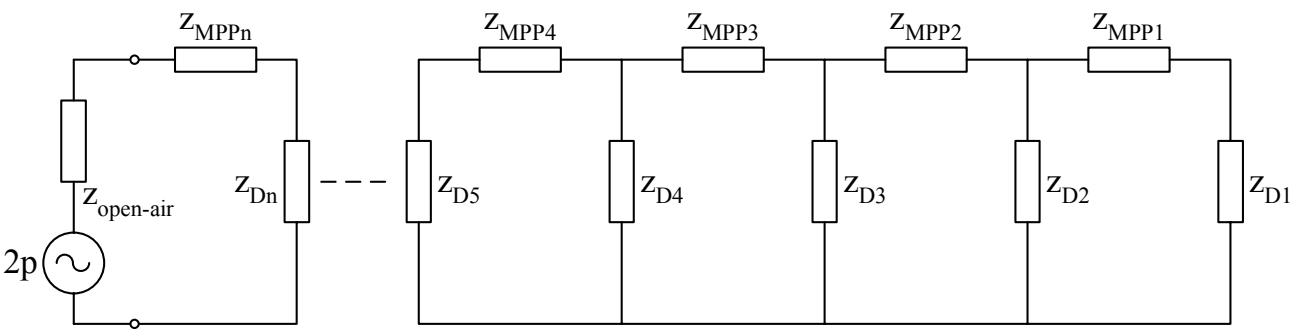

Figure 2. Electro-acoustic analog circuit

Using electrical analog circuit, the double leaf MPP absorber can be extended to $n$ number of panels. The total impedance of $n$ leaf MPPs at the surface of MPP ${ }_{n}$ will be

$$
\left\{\begin{array}{l}
z_{r+1}=\frac{z_{r} z_{D(r+1)}}{z_{r}+z_{D(r+1)}}+z_{m p p(r+1)}, \quad r=(1,2,3, \ldots, n-2, n-1) \\
z_{1}=z_{m p p 1}+z_{D 1} .
\end{array}\right.
$$

In this type of configurations, the effect of sound transmission loss has to be taken into consideration because the terminating wall does not exist. The sound transmission loss due the absence of the terminating wall is given by the following approximation, based on the electrical circuit depicted in Fig. 2. The sound transmission loss is determined by the loss in $\mathrm{Z}_{D 1}$ that is

$$
T L_{r+1}=\left[\frac{2}{z_{r+1}+1} \cdot \frac{z_{D r+1}}{z_{D r+1}+z_{m p p r}+z_{r}} \cdots \frac{z_{D 2}}{z_{D 2}+z_{1}}\right]^{2}, \quad r=(1,2,3, \ldots, n-2, n-1) .
$$

From the total impedance given by Eq. (11), the sound absorption coefficient of multi-leaf MPP without including the sound transmission loss is expressed by

$$
\alpha_{\text {multi-leaf MPP without TL }}=1-\left|\frac{z_{r+1}-1}{z_{r+1}+1}\right|^{2} \text {. }
$$

Finally, the overall sound absorption coefficient of a multi-leaf microperforated panel by including the sound transmission loss is expressed by

$$
\alpha_{\text {true }}=\alpha_{\text {multi-leaf MPP without TL }}-T L_{r+1} \text {. }
$$

\section{Numerical results and discussions}

The performance of multi-leaf microperforated panel absorber for different configuration is discussed below. The proposal to get optimum absorption coefficient is also discussed based on the order of panel with uniform or varied perforation diameter and air spaces. In all of the following studies, the panel thickness $(t)$ of $1 \mathrm{~mm}$, the distance between holes $(b)$ of $3 \mathrm{~mm}$, the sound speed (c) of $340 \mathrm{~m} / \mathrm{s}$, the air density $\left(\rho_{o}\right)$ of $1.2 \mathrm{~kg} / \mathrm{m}^{3}$, and the air viscosity $(\eta)$ of $17.89 \mu \mathrm{Pa}$.s are used. 


\subsection{Double-leaf MPP}

The study is started from two-leaf MPP with diameters of $0.3 \mathrm{~mm}, 0.35 \mathrm{~mm}, 0.4 \mathrm{~mm}$, $0.45 \mathrm{~mm}$ and $0.5 \mathrm{~mm}$ with holes' separation distance of $3 \mathrm{~mm}$. These values correspond to the perforation ratio of $0.79 \%, 1.07 \%, 1.4 \%, 1.77 \%$ and $2.18 \%$ respectively. The distance between two MPPs was set from $10 \mathrm{~mm}$ to $60 \mathrm{~mm}$. The first investigation is carried out with two identical MPPs. The results of these arrangements are depicted in Fig. 3.
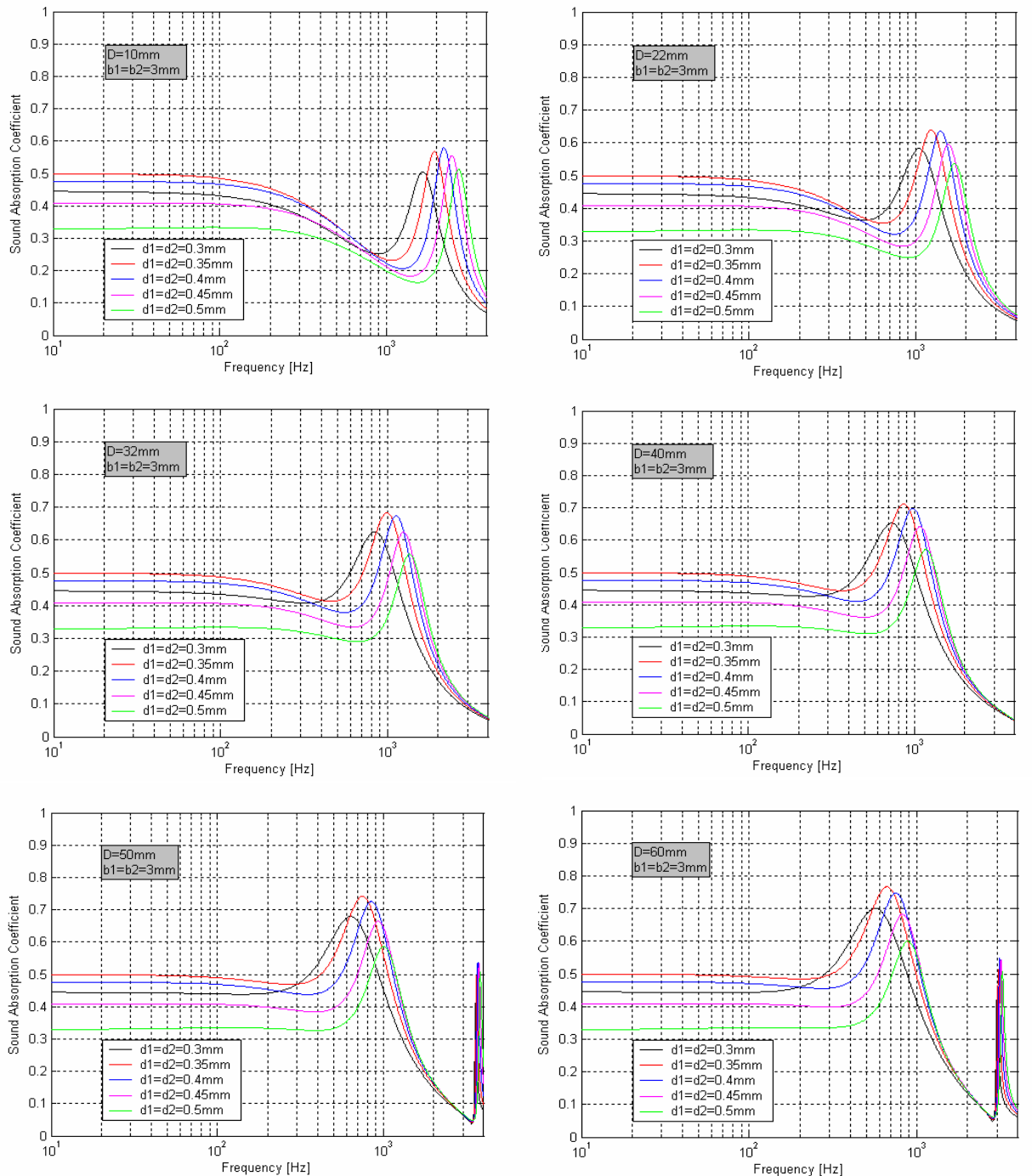

Figure 3. Effect of air space's separation on sound absorption of two-leaf MPP

One can observe from Fig. 3 that two-leaf MPP will give a quite high (up to 0.5) value of sound absorption coefficient at low frequency region. This fact is an advantage of this type of MPP absorber compared with the conventional one. It is also observed that the value of sound absorption coefficient will increase with the increase of the distance between panels but the peak of sound absorption coefficient will be shifted to lower frequency region. In the numerical examples above, sound absorption coefficient will increase when the hole's diameter of the panel increases from $0.3 \mathrm{~mm}$ to $0.35 \mathrm{~mm}$. After that the value will be decreasing correspondingly. In this case, the optimum value of absorption can be obtained with diameter of holes $0.35 \mathrm{~mm}$ with distance between holes of $3 \mathrm{~mm}$, independent on the value of distance between panels.

Sound absorption coefficient when the two MPPs are not identical is shown in Fig. 4. Figure 4a shows the results when the left-hand side panel (MPP2) is kept constant with 
diameter of $0.3 \mathrm{~mm}$ meanwhile the right-side panel (MPP1) varies in diameter from $0.3 \mathrm{~mm}$ to $0.6 \mathrm{~mm}$. The distance between panels is set the same, which is $50 \mathrm{~mm}$.

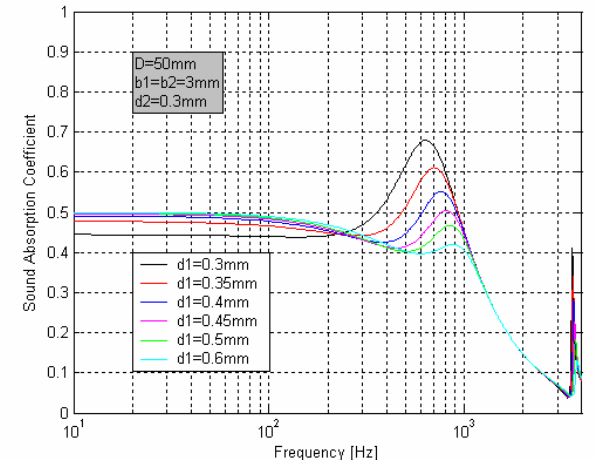

(a)

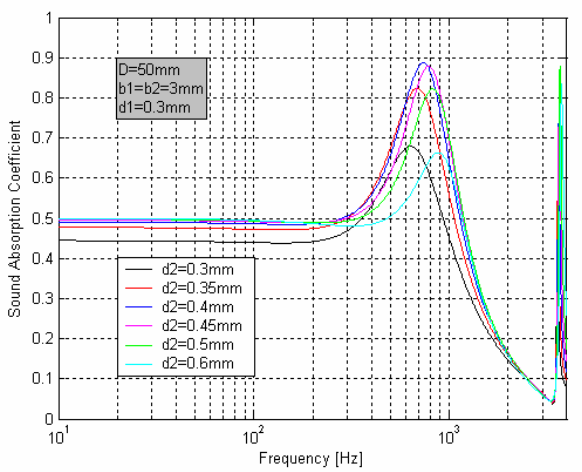

(b)

Figure 4. Effect of panels' arrangement on sound absorption of two-leaf MPP

The result of the opposite arrangement of the two panels, i.e. MPP1 is kept constant and varied MPP2 is shown in Fig. 4b. It is noticed that the order of panel plays important role to get optimum sound absorption coefficient. In general, to get a better sound absorption coefficient, the panel with higher perforation ratio has to be placed close to the noise source. These phenomena can be explained by the fact that if the right-hand side panel has smaller perforation ratio, it will give higher impedance for the sound to pass through the panel resulted in smaller sound transmission loss, i.e. higher sound absorption coefficient.

\subsection{Three-leaf MPP}

For three-leaf MPP, the investigation is firstly carried out with all three identical MPPs (MPP1=MPP2=MPP3). Here the effect of the order of the air distance between panels is studied. The distance between MPP3 and MPP2 (D3) and the distance between MPP2 and MPP1 (D2) are interchanged while the total distance is maintained the same or constant. The results of such configurations are shown in Fig. 5. Figure 5a shows the results when the distance of panels closer to the noise source (D3) is constant and that one far from noise source (D2) is varied. The results of the opposite arrangement are depicted in Fig. 5b.

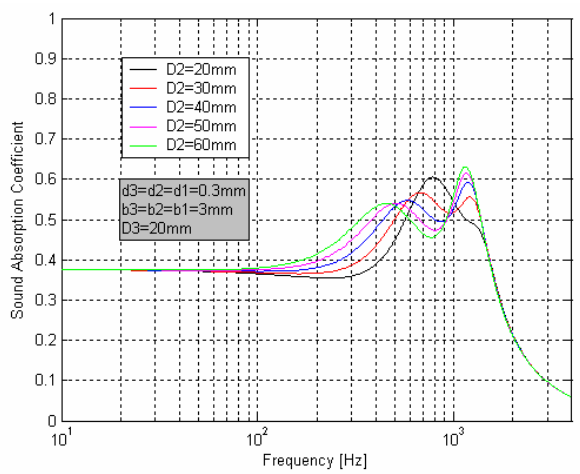

(a)

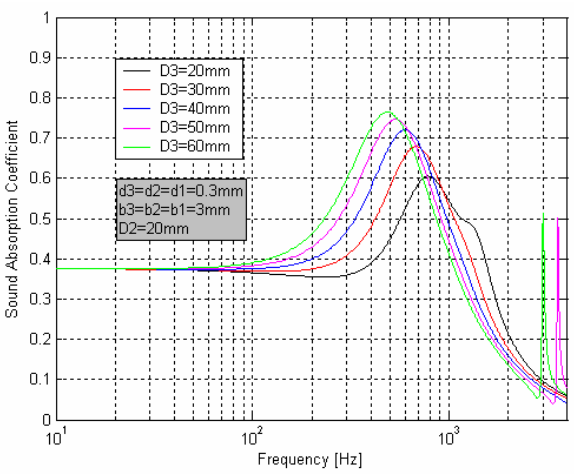

(b)

Figure 5. Effect of airspace's order on sound absorption of three-identical-leaf MPP

It can be noted from Fig. 5 that the order of airspace between MPPs does affect the value of sound absorption coefficient. If the air space that is closer to noise source (D3) is smaller than that far from the noise source (D2) or D3 $<$ D2 (see Fig. 5a), the peak value of absorption will be smaller compared with the opposite arrangement but it has wider 
frequency band. The sound absorption of the opposite arrangement (D3>D2) will give higher peak of sound absorption over lower frequency region, but the frequency band is narrower. It is also noticed that overall sound absorption shifted to lower frequency region.

In Fig. 6, three panels with all possible arrangements of panels are depicted. Figure 6a and Fig. $6 \mathrm{~b}$ show the results for the total width of $60 \mathrm{~mm}$ and $40 \mathrm{~mm}$ respectively.

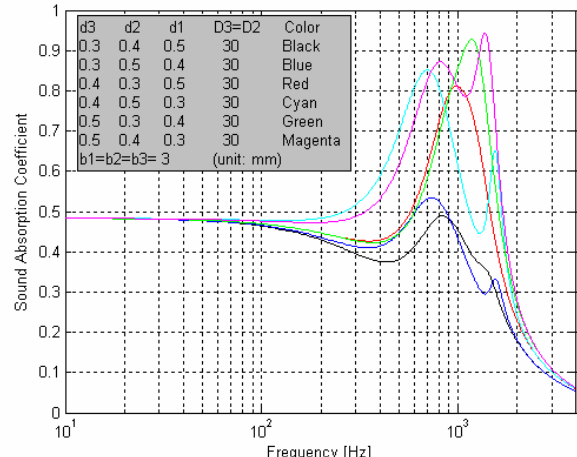

(a)

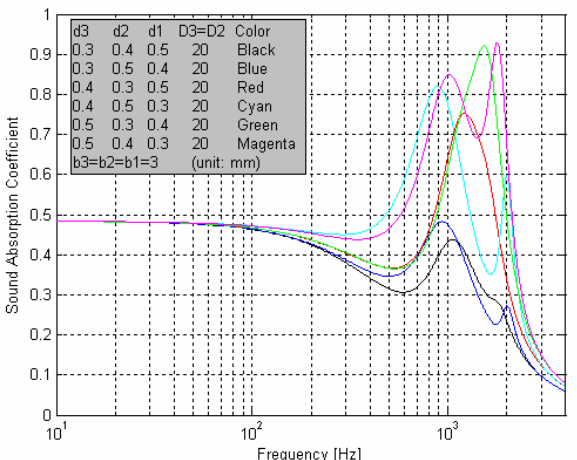

(b)

Figure 6. Effect of panels' arrangement on sound absorption of three-different-leaf MPP

Both figures show that in order to get the best value of sound absorption the order of the panels, in term of hole's size or perforation ratio, should be in "big-smaller-smallest" order or $\mathrm{d} 3>\mathrm{d} 2>\mathrm{d} 1$. The reason of these phenomena is the same with the case of double-leaf MPP. In addition, the shift in frequency region is also noticed which is the same with the phenomena found in the conventional arrangement of MPP.

\subsection{Four-leaf MPP}

The panels used for four-leaf MPP have diameter of $0.3 \mathrm{~mm}, 0.4 \mathrm{~mm}, 0.5 \mathrm{~mm}$ and $0.6 \mathrm{~mm}$. All possible arrangements of these panels were investigated. The total distance between panels is set constant of $60 \mathrm{~mm}$ and the same for each adjacent panel, which is $20 \mathrm{~mm}$. From four-leaf MPP, there are 24 possible arrangements of panels. In Fig. 7, two curves correspond to the configurations that give optimum and minimum sound absorption coefficients are shown.

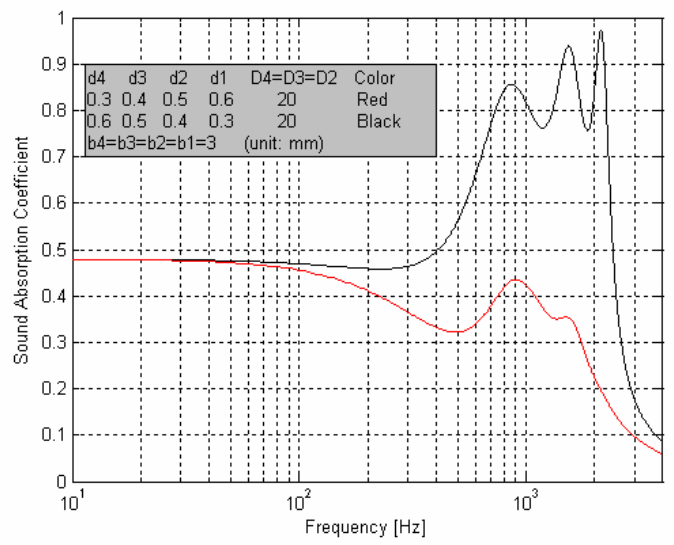

Figure 7. Effect of panels' arrangement on sound absorption of four-different-leaf MPP

The optimum sound absorption can be achieved with the same method shown in three-leaf MPP, i.e. by putting the panel with biggest diameter (highest perforation ratio) close to noise source and the next remaining panels should be smaller and smaller in diameter $(\mathrm{d} 4>\mathrm{d} 3>\mathrm{d} 2>\mathrm{d} 1)$. All other-possible arrangements will give various results but the results are still within the two curves shown in Fig. 7. 
Meanwhile, in Fig. 8, the effect of airspace between panels is presented. Figure 8a shows the result for the panels with diameter varies from $0.6 \mathrm{~mm}$ to $0.3 \mathrm{~mm}$ (the same with the arrangement of panels that give the best value as shown in Fig. 7), and Fig. 8b shows the result for uniform $(\mathrm{d} 4=\mathrm{d} 3=\mathrm{d} 2=\mathrm{d} 1=0.3 \mathrm{~mm})$ panels.

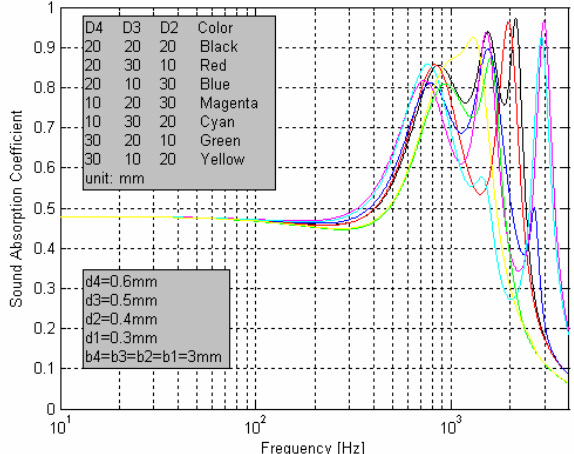

(a)

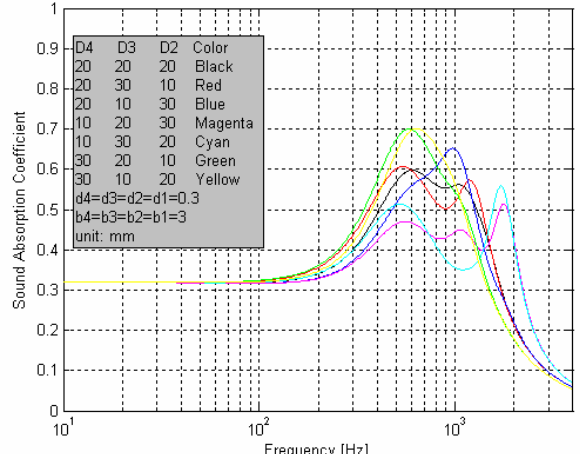

(b)

Figure 8. Effect of airspace's order on sound absorption of four-leaf MPP

One can observe that the order of airspace give almost the same effects as shown for three-leaf MPP. The results from other panels' size also show the same effect. From Fig. 8b one can clearly notice the effect of airspace's order on sound absorption coefficient. If the airspace closer to the noise source is bigger than the remaining airspaces, i.e. D4 $>$ D3 $>$ D2, the peak of sound absorption (shown with green line) will be higher with the compensation of a narrower frequency band. Conversely, the airspace with order of $\mathrm{D} 4<\mathrm{D} 3<\mathrm{D} 2$, the sound absorption peak will be lower (shown with magenta line) but it has wider frequency band. As can be seen from Fig. 8a, in the frequency range up to $2500 \mathrm{~Hz}$, the optimum sound absorption, i.e. high value of absorption, can be achieved when the distances between panels are the same (D4=D3=D2) and the arrangement of panels from left to right is in order of $\mathrm{d} 4>\mathrm{d} 3>\mathrm{d} 2>\mathrm{d} 1$ (as shown with black line).

From all of the calculation results presented above, the sound absorption coefficient at quite low frequency region is observed. The reason for this phenomenon is the absence of the solid terminating wall. This phenomenon, the low frequency absorption, was also found and reported in the conventional MPA $[1,2,3]$, when one has MPP with solid backing panel that located at far distance, e.g. $\mathrm{D}>1 \mathrm{~m}$, from the panel resulted in sound absorption at low-mid frequency region.

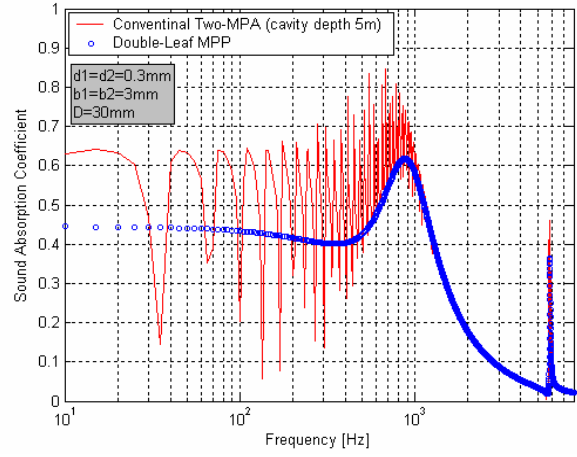

(a)

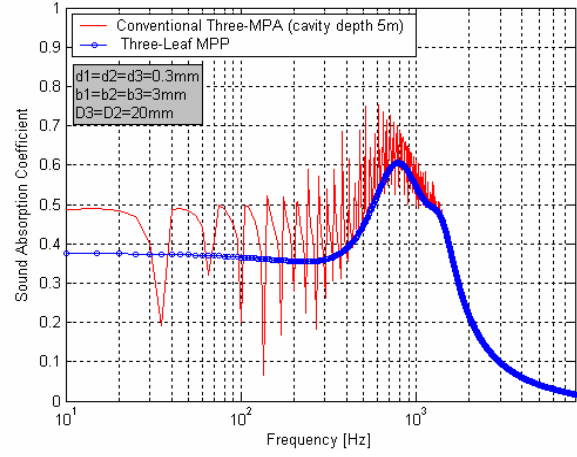

(b)

Figure 9. Comparison of sound absorption between leaf MPP and conventional MPA

Figure 9 shows a comparison between leaf-MPP absorber and conventional MPP absorber (with a solid backing panel at long distance). As can be seen from Fig. 9, the absorption at low-mid frequency region obtained from leaf-MPP arrangement is almost the same with the average value of that obtained from the conventional MPA. 


\section{Experimental results}

In the experiment investigation, MPP panels are made from plastic (polyester). In this experiment, three MPP panels are made. The first two MPPs have the same perforation diameter of $0.3 \mathrm{~mm}$ and the third MPP has perforation diameter of $0.5 \mathrm{~mm}$. All the three MPPs have the same separation distance between holes of $3 \mathrm{~mm}$. The panel thickness used is $1 \mathrm{~mm}$. These control parameters were selected so that the measurements results can be compared with the calculation results shown in Fig. 3 and Fig. 4 in Section 4. The measurement was carried out using an impedance tube. Transfer function method was implemented for the sake of simplicity. In this validation experiment, the frequency range coverage is from $400 \mathrm{~Hz}$ to $1800 \mathrm{~Hz}$ based on the dimension of impedance tube used in this measurement [10]. Firstly, the experiment with two-identical leaf-MPP was carried out. Figure 10a and Fig. 10b show the results of two-identical-leaf MPP with $22 \mathrm{~mm}$ and $32 \mathrm{~mm}$ separation distance between panels, respectively. It can be observed that a quite good agreement was found between the measurements results and the numerical calculation results.

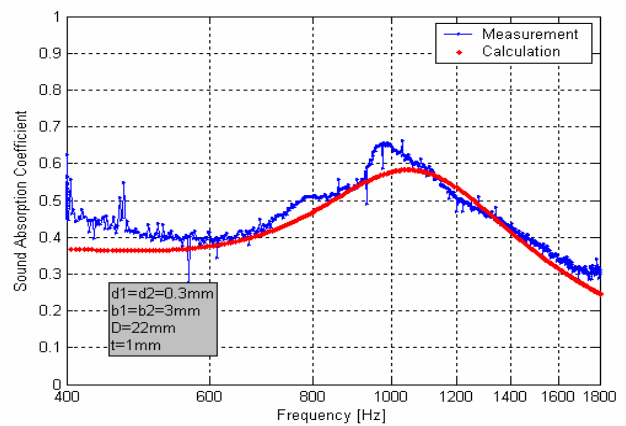

(a)

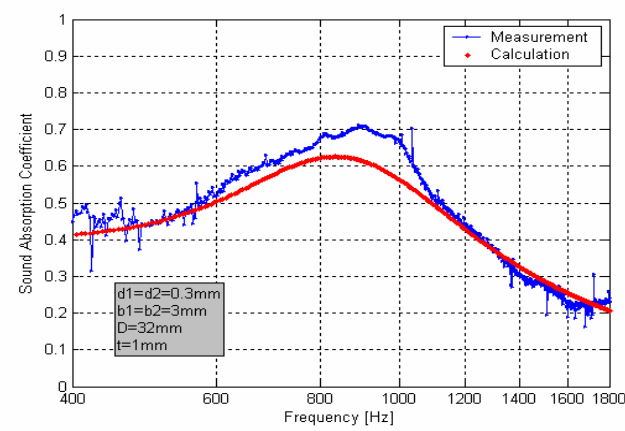

(b)

Figure 10. Measured and calculated sound absorption for two-identical-leaf leaf MPP

The experiments were then extended with two-leaf MPP but with different size of holes or diameters. Figure 11a shows the results when the panel closer to the sound source has diameter of $0.3 \mathrm{~mm}$ and the panel far from the sound source has diameter of $0.5 \mathrm{~mm}$. Meanwhile the results of the opposite arrangement, i.e. panels' position is switched or interchanged, is shown in Fig. 11b. As can be observed, again a quite good agreement was found between the measurements results and the numerical calculation results. Nevertheless, with these four figures, it is shown that the experimental works well to verify the numerical calculation appropriately.

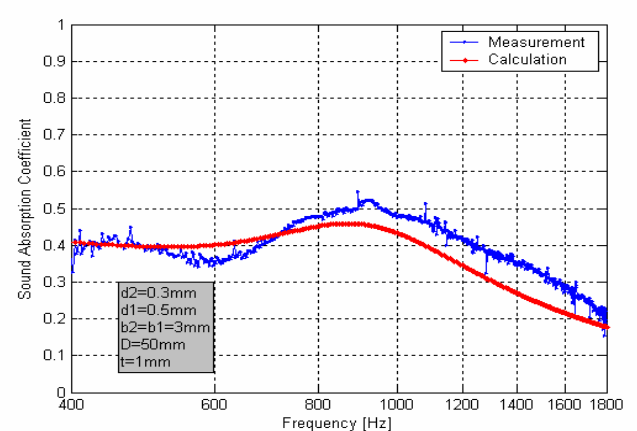

(a)

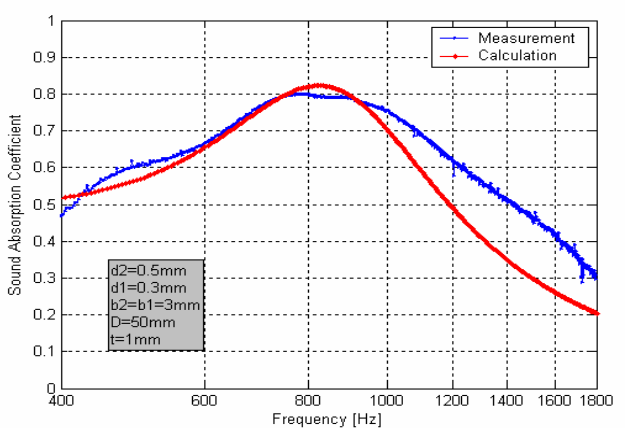

(b)

Figure 11. Comparison of sound absorption for two-leaf MPP with switched panels' position 


\section{Conclusions}

Multi-leaf MPP has been studied both theoretically and experimentally. The studies proved that multi-leaf MPP (MPP without solid backing panel) can give additional sound absorption especially in low frequency region in addition to the clear peak as given by the conventional one. The optimum absorption coefficient in terms of high value and wider frequency range can be achieved by putting panel with bigger perforation ratio closer to noise source. The orders of the cavity between panels also affect the sound absorption, but the effect is not as significant as the order of the panel does. It was also found that there is no one single parameters that can give the optimum value of sound absorption because it seems that there is the optimal value for each single parameters but cannot be combined to get the overall optimum sound absorption. There will be always a drawback found, such as, wider frequency band but a lower value of absorption and vise versa and also the shift in the frequency region. However, in this study, the guidance to get the best value of absorption coefficient has been pointed out. This type of absorber can have an advantage when the attenuation of lower frequency noise is also of interest. Since a multi-leaf MPP can work effectively as sound absorber for sound incidence from both sides, it can be used effectively as a space absorber, e.g., as suspended absorber or as a working space partition in offices, workshops or hospitals. In many engine enclosures, when a heat release is also a concern in addition to the noise attenuation, this type of absorber will be of help. This type of absorber could be applicable also for tunnel partition on the track of subway train or partition wall for highway since it can absorb noise from both sides of roads.

\section{References}

[1] D.Y. Maa, "Theory and design of microperforated-panel sound-absorbing construction," Scientia Sinica, Vol. XVIII (1975), pp. 55-71.

[2] D. Y. Maa, "Microperforated-Panel Wideband Absorbers," Noise Control Engineering Journal, Vol. 29, No. 3 (1987), pp. 77-84.

[3] D.Y. Maa, "Potential of Microperforated Panels Absorber", Journal of the Acoustical Society of America, Vol. 104, No. 5 (1998), pp. 2861-2866.

[4] Zhang Z.M., and Gu X.T., "The Theoretical and Application Study On A Double Layer Microperforated Sound Absorption Structure", Journal of Sound and Vibration, Vol. 215, No. 3 (1998), pp. 399-405.

[5] Kang J., and Fuchs H.V., "Predicting The Absorption of Open Weave Textiles and Micro Perforated Membranes Backed By An Air Space", Journal of Sound and Vibration, Vol. 220, No.5 (1999), pp. 905-920.

[6] Thomas C., Weltin U., and Petersen S., "Sound Transmission Loss of Double-Panel Partitions with Internal Microperforated Panel Absorber", Proceeding of the $10^{\text {th }}$ International Congress on Sound and Vibration, (2003).

[7] Cobo P., Pfretzschner J., Cuesta M., and D.K. Anthony, "Hybrid Passive-Active Absorption Using Microperforated Panels", Journal of the Acoustical Society of America, Vol. 116, No. 4 (2004), pp. 2118-2125.

[8] Congyun Z., and Qibai H., "A method for calculating the absorption coefficient of a multi-layer absorbent using the electro-acoustic analogy", Journal of Applied Acoustics, Vol. 66, No. 7 (2005), pp. 879-887.

[9] Sakagami K., Morimoto M., and Koike W., "A Numerical Study of Double-leaf Microperforated Panel Absorbers", Journal of Applied Acoustics, Vol. 67, No. 7 (2006), pp. 609-619.

[10] Cox T.J., and D'Antonio P., "Acoustic Absorbers and Diffusers: Theory, design and application", Spon Press, Taylor \& Francis Group, Chapter 3(2004), pp. 58-85. 